\title{
Auditory screening of special care neonates using the auditory response cradle
}

\author{
B McCORMICK, D A CURNOCK, AND F SPAVINS \\ Nottingham General Hospital and City Hospital, Nottingham
}

SUMMARY The Linco-Bennett auditory response cradle is a microprocessor controlled device for screening the hearing of neonates. A total of 396 neonates admitted to a special care unit were tested on the cradle and later followed up in a comprehensive test programme between the ages of 3 months and 8 months. Altogether $374(94 \%)$ were available for follow up. The use of the cradle resulted in the detection of six neonates with appreciable deafness. One neonate who passed the cradle test has severe bilateral hearing impairment. The false alarm rate for neonates failing two tests on the cradle but having normal hearing at follow up was $4 \cdot 3 \%$.

The auditory response cradle was designed for use in mass screening programmes but testing the hearing of all newborns would require many staff. It is argued that this is unrealistic when resources are scarce, but that neonates in high risk groups should have their hearing screened at birth by an objective test such as this. The cradle has considerable potential but its method of use and the 'decision making' programme could be improved.

It is important that hearing impairment in babies should be detected and remedial action taken for those affected as early as possible. In most cases the specific objective is fitting hearing aids at the earliest possible age, and we now fit aids to babies as young as 10 weeks. Delaying the provision of amplification to hearing impaired babies may lead to disrupted social, emotional, intellectual, and linguistic development, and some of the devastating effects may be only partially reversed by subsequent programmes of remedial treatment. ${ }^{1-4}$

It has long been known that newborn babies react to sound by responses such as startle, head turn, and change in respiratory pattern. Previously, however, it has not been possible to use these responses as the basis of an objective test of hearing in newborns because of generally high degrees of spontaneous activity in the awake baby and because of observer bias. The auditory response cradle was developed by Dr M J Bennett of Brunel University over an eight year period as an automatic, microprocessor controlled device to detect these hearing responses against a background of spontaneous activity and without observer bias. ${ }^{5}$

The results of long term follow up of newborns tested with the cradle have recently been published ${ }^{11}$ and the present paper extends the scope of the evaluation of the auditory response cradle b. $y^{+}$ concentrating on neonates requiring special care.

Nottingham City Hospital was the first centre to receive an auditory response cradle for an evaluation trial on special care babies. The decision to use the cradle on these babies was taken for two reasons. Firstly, no auditory response cradle trial had then been undertaken on this group, and it was not known whether the cradle would be as suitable for these babies as it was for the normal term babies used in the development phase.$^{6-8}$ Secondly, it has been well documented that the incidence of hearing disorders in low birthweight and special care babies is much higher than in normal term babies. ${ }^{910}$ Effective screening of an at risk group would, therefore, identify a good percentage of all hearing impaired babies.

\section{Auditory response cradle}

A full description of the auditory response cradle may be found elsewhere. ${ }^{5-8}$ Briefly, the unit consists of a trolley, of a similar size to a normal neonatal 2 cradle, which houses a microprocessor and the 0 associated electronics beneath a moulded plastic $\mathbb{\Phi}$ cradle. The microprocessor records and stores in- $\stackrel{?}{\rightarrow}$ formation from a series of non-invasive, pressure 
activated transducers. These monitor the baby's head turning, backward head jerk or startle responses, body activity, and respiration movements at times when sound stimuli are presented, and also during 'no sound' intervals (blank trials). The auditory response cradle 'passes' or 'fails' a child by referring to a decision table which compares the numbers of responses to sound trials and to blank trials. The preprogrammed decision criteria were set by the manufacturer after trials on healthy, normal birthweight babies.

\section{Subjects and methods}

Over a one year period from July 1981 to June 1982, 396 patients from the neonatal special care unit of the City Hospital (representing $56.6 \%$ of the total admissions to the unit during this time) were tested in the auditory response cradle. The total unit admissions constituted $5.7 \%$ of the total birth population in the hospital. Retrospective analysis of the distributions of age, birthweight, and sex in the tested and non-tested special care groups confirmed that it was a representative sample (Table). Some of the babies admitted during this period could not be tested because of transfer back to referring hospitals as soon as intensive care was no longer required, or because of short stay on the unit, or very occasionally, because of faults in the cradle or shortage of staff.

Permanent records were made of each neonate's head turn, body activity, startle response, and breathing response during sound and blank intervals, using the display facility on the control panel. This thorough approach later provided the necessary data to evaluate the validity of the criteria incorporated into the decision algorithm giving the manufacturer's automatic 'pass' or 'fail' outputs.

Neonates who failed the first test in one or both ears were retested at least one day later. The mean time between tests was 4.5 days (SD 4.5 , range 0 to 17 ) and the mean gestational age at first testing was 37 weeks (SD 2.9, range 30 to 50 weeks). Testing was undertaken when the baby was asleep or in a quiet and settled state, usually in the period from one hour after a feed to one hour before the next.

Table Comparison of tested and untested groups

\begin{tabular}{|c|c|c|c|c|}
\hline & \multirow{2}{*}{$\begin{array}{l}\text { Birthweight } \\
(g) \\
\text { Mean (sd) }\end{array}$} & \multirow{2}{*}{$\begin{array}{l}\begin{array}{l}\text { Gestational age } \\
\text { (weeks) }\end{array} \\
\text { Mean (sd) }\end{array}$} & \multicolumn{2}{|l|}{$\begin{array}{l}\text { Sex } \\
(\%)\end{array}$} \\
\hline & & & Boy & Girl \\
\hline Tested group & $2224 \quad(754)$ & $35 \cdot 1(3 \cdot 5)$ & 59 & 41 \\
\hline Untested group & $2556(1031)$ & $35 \cdot 6(4 \cdot 7)$ & 56 & 44 \\
\hline
\end{tabular}

\section{Follow up test programme}

A thorough follow up of all of the tested babies was planned with the intention of verifying the eventual hearing status of every baby. The timing and content of the follow up was tailored according to the auditory response cradle test results and the presence of high risk factors. A list of risk criteria for hearing impairment was drawn up and applied to each baby. This included: (1) the recording of complications including fetal distress, apnoeic attacks needing intubation (with or without ventilation), oxygen therapy, ventilation, jaundice sufficient to need bilirubin measurement, rhesus haemolytic disease, exchange blood transfusion, meningitis, hydrocephalus, fits, and administration of a potentially otoxic drug; (2) recording the presence of congenital abnormalities including abnormal appearance of face or jaws, other congenital abnormalities, and whether congenital infection screen performed; (3) recording maternal history including any family history of hearing impairment from infancy, maternal rubella contact with significantly raised titres, severe maternal illness in pregnancy threatening the fetus (for example fits, unconsciousness), and administration of an otoxic drug in pregnancy.

All babies who failed the auditory response cradle test and all of those with three or more risk criteria or with a family history of deafness were asked to come back to the specialist Children's Hearing Assessment Centre at the General Hospital for follow up. Follow up included distraction (orientation response) testing between the ages of 3 and 8 months, together with middle ear impedance measurement and recording of the stapedial reflex, auropalpebral reflex, and startle response. Those who had failed the cradle test twice had brainstem evoked response audiometry when six weeks beyond the expected date of delivery and then full follow up at the hearing centre.

Those who passed on the cradle and had fewer than three risk criteria, or none at all, were followed up in the community at 6 to 8 months of age by health visitors who had recently been trained by one of the authors (BMc). The results of the effectiveness of the health visitors' testing in the Nottinghamshire area have been published elsewhere. ${ }^{12}$

\section{Results}

Fig. 1 summarises the results for the 396 babies tested on the auditory response cradle. Through the combined use of the cradle and the follow up programme, three children with bilateral and three 


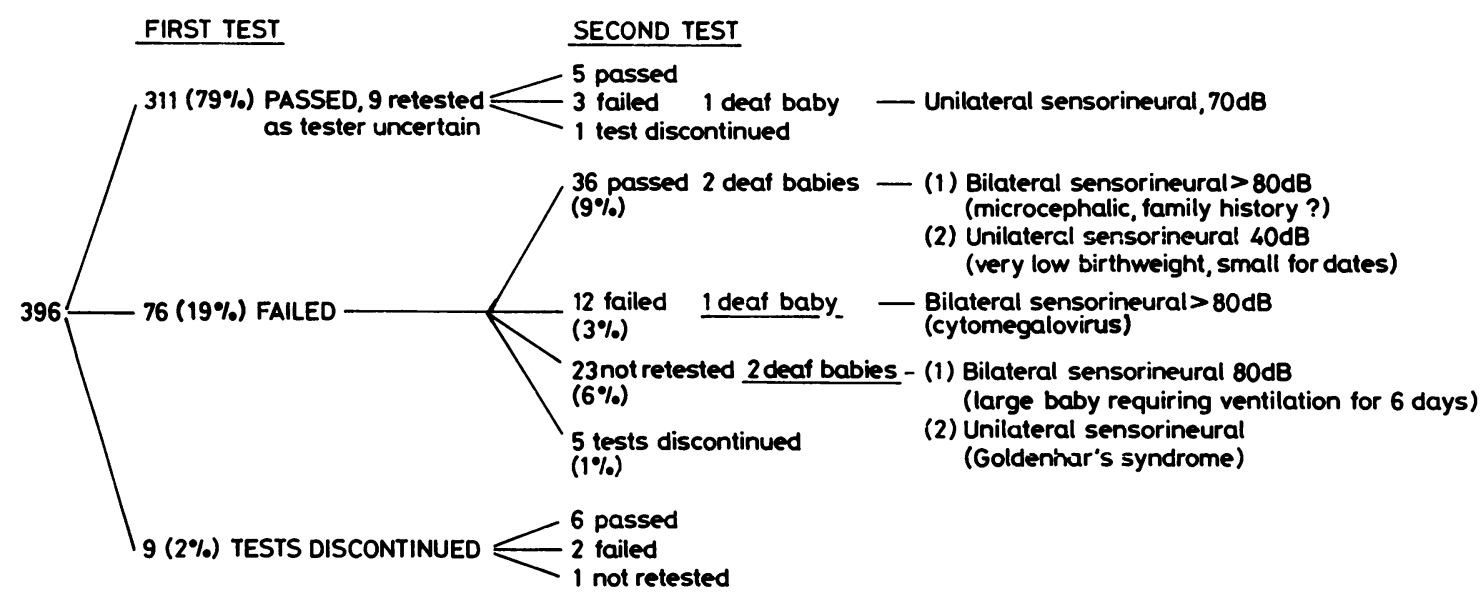

Fig. 1 Auditory response cradle-outcome in 396 infants.

with unilateral sensorineural losses have been identified.

It can be seen from Fig. 1 that five of the six hearing impaired neonates failed the first cradle test and were, therefore, correctly identified at that stage. Of the 62 very low birthweight neonates (1500 $\mathrm{g}$ or less) tested, one was found to have a unilateral sensorineural loss. One baby with an appreciable unilateral loss passed the first test and would not have been identified by the cradle. This baby, however, was retested along with several others that the tester considered had made very poor responses, and indeed she failed the second test. The false-positive rate at each stage of testing was approximately $20 \%$. This is higher than the $5 \cdot 3 \%$ reported by Bennett and Wade, ${ }^{11} 13$ as would be expected for special care babies. Three of four hearing impaired neonates who were tested twice passed the test on one of the two occasions. This may be related to the shortcomings of decision rule and will be discussed in more detail later. The two remaining neonates with sensorineural losses, identified at follow up, failed the first test but were discharged before a second test could be carried out. Therefore, only one hearing impaired neonate actually failed the auditory response cradle test on two occasions.

According to the follow up programme, 209 (53\%) neonates were scheduled to attend the Children's Hearing Assessment Centre for further hearing tests. In fact, $157(40 \%)$ actually attended. The remaining $52(13 \%)$ having moved, died, refused, or defaulted their appointments. The 'take up' rate for babies listed to attend the centre was therefore $75 \%$. In all, health visitor routine screen-

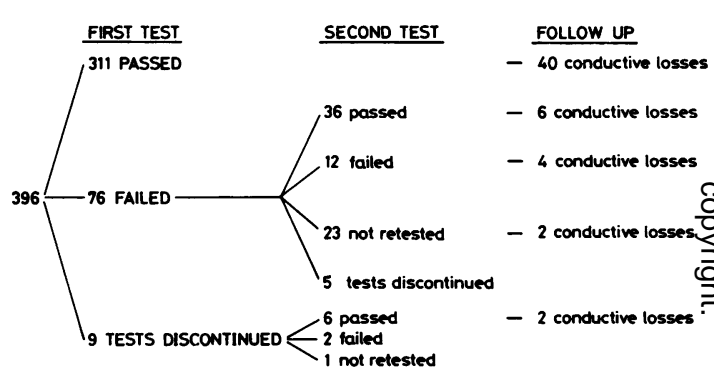

Fig. 2 Conductive losses found at hearing assessment centre follow up.

ing results were requested for 239 babies. At the time of writing 22 results have not yet been traced; 19 from the group who passed the first test, and three who failed the first test but passed the second test. This is mainly due to difficulties in collecting information on babies who have moved from the area, or changed their names or general practitioner, or both.

At the time of initial follow up, 54 infants were found to have a temporary conductive hearing loss which may or may not have been present at the time of testing on the cradle (Fig. 2). Four of these have since been listed for operation. Owing to the intermittent nature of conductive hearing problems, it is difficult to determine how many infants were affected at some time during the programme.

\section{Discussion}

In this study a considerable amount of information 
has been collected on the performance of neonates in the auditory response cradle. The immediate focus of interest is how successful the cradle was in identifying hearing impaired babies in special care. The cradle failed to identify one patient with bilateral sensorineural deafness and two with unilateral sensorineural losses in one of their pair of tests. These false negative findings raised concern and indicated that the cradle's decision criteria might not be wholly appropriate. The criteria have been studied in detail by Davis, ${ }^{14}$ using data obtained in our study, and he concludes that the stringence of the decision criteria in the auditory response cradle is variable across the test sequency and not fixed as is stated by Bennett and Wade. ${ }^{13}$ Davis suggests improvements to the statistical decision criteria which should lead to an all round improvement in the cradle's performance. Nevertheless, the technique seems to have potential: with the incorporation of improved decision criteria and by always devoting a complete test sequence to each ear separately, the cradle should provide an effective neonatal screening device for special care and at risk neonates.

The auditory response cradle was designed for use in mass screening. Given the limited time and staff resources available, the decision to concentrate attention on special care babies because of the anticipated higher prevalence of hearing loss in that group seems to be justified. For example, to reach the present interim conclusions, favourable to the cradle, would have required a trial of approximately 10 times the length of the present one in an unselected population of neonates.

The prevalence figure for bilateral hearing loss here has been found to be three in 396 or 7.6 per 1000 births $(95 \%$ confidence interval $0-16)$. This is approximately 10 times greater than that expected for the newborn population as a whole. ${ }^{15}$ The prevalence for unilateral and bilateral hearing loss combined has been found to be six in 396 or $15 \cdot 2$ per 1000 (95\% confidence interval 3-27).

The prevalence of sensorineural hearing loss in the very low birthweight babies was one in 62 . This figure is much lower than the $9 \%$ quoted by Abramovich et al ${ }^{10}$ from University College Hospital but their babies were born during the years 1966 to 1972 and since that time further improvements have been made in ventilation and intensive care methods.

The time taken to test each neonate is an important consideration for any screening programme. The manufacturers quote a test time of 3 to 10 minutes for their recommended method, applied binaurally to babies of normal birthweight. ${ }^{13}$ In the present study the mean test time in a sequential sample of 66 tests was 15 minutes per ear (range 3 to 45 minutes). The test time could be halved by testing bilaterally and accepting that unilateral hearing losses have a lower probability of being detected. This could lead to false assurances being offered to parents about the integrity of their children's hearing. It is concluded in this study and in that of Davis $^{14}$ that each ear should be tested separately by allocating a full test sequence to each ear; which not only affords some possibility of detecting a unilateral condition, but gives a more thorough test for a bilateral condition.

As a general policy, when resources are limited it is clearly sensible to concentrate initially any expensive high technology resource with special staffing requirements on a high risk group. This study has shown that it is possible to use the auditory response cradle with very low birthweight and other special care babies. Having satisfied this as the priority need, and assuming that this high technology resource is not available for every baby, the remaining more scattered population of neonates could be screened by using a questionnaire handout to parents of the type advocated by McCormick, ${ }^{12}$ McCormick et al, ${ }^{16}$ Latham and Haggard, ${ }^{17}$ and Mahoney and Eichwald. ${ }^{18}$ These investigators and others have shown that parents' suspicions of hearing difficulties in their offspring are reliable indicators of true impairments and that these suspicions can be systematically focussed by offering parents a handout which lists the expected responses to everyday sounds at home. Medical parsimony and political egalitarianism will certainly constitute obstacles to the acceptance of truly economic adaptations of screening systems such as a two tiered structure, especially as they seem more complicated at the outset. In the context of screening 5 year old children for otitis media, Haggard et al ${ }^{19}$ have likewise argued that the overall requirements of the screening are best met not by a single screen, but a two tiered approach. Here the placing of a child on an at risk register or the evident need for intensive care, constitutes the first tier; we suggest that the second tier be conditional upon the first, that is cradle testing for those at risk and a community distributed leaflet for others. At a time when no routine systematic hearing screening programme exists for neonates the suggested division may offer the only workable and affordable framework for health authorities who wish to screen effectively for hearing impairments in children. Encouraged referrals, in turn, imply a unit with staff especially trained for assessing the very young. District health authorities should have such facilities but not all do at present.

We conclude that at the initial stage of field trial 
evaluation the auditory response cradle has potential for the very early detection of hearing impairment in special care babies and that its reliability should be improved with the incorporation of a better decision algorithm.

We thank Dr A Davis and Professor M P Haggard of the MRC Institute of Hearing Research and Professor D Hull for their support and helpful comments. The study could not have been undertaken without the long term support of the nursing staff, and we thank M Kendall, J Foster, R Haddow, A Turner, S Wyatt, and P Hale for arranging the testing programme and for administering the tests.

\section{References}

${ }^{1}$ Elliott LL, Armbrusher VB. Some possible effects of the delay of early treatment of deafness. $J$ Speech Hearing Res 1967;110:209-24.

2 Tervoort B. Development of language and critical periods in the young deaf child: identification and management. Acta Otolaryngol (Stockh) 1964;206:247-54.

3 Brothman R, Orr N. Value of screening for deafness in first year of life. Arch Dis Child 1978;53:570-3.

${ }^{4}$ Gerber SE, Mencher GT. (eds). Early diagnosis of hearing loss. New York: Grune and Stratton, 1978.

5 Bennett MJ. The auditory response cradle: a device for the objective assessment of auditory state in the neonate. Symposia of the Zoological Society of London 1975;37:291-305.

6 Bennett MJ. Trials with the auditory response cradle Ineonatal responses to auditory stimuli. $B r \quad J$ Audiol 1979;13:125.34.

7 Bennett MJ. Trials with the auditory response cradle II-the neonatal respiratory response to an auditory stimulus. $\mathrm{Br} J$ Audiol 1980;14:1-6.

${ }^{8}$ Bennett MJ. Trials with the auditory response cradle III-head turns and startles as auditory responses in the neonate. $\mathrm{Br} J$ Audiol 1980;14:122-31.
${ }^{9}$ Simmons FB, McFarland WH, Jones FR. An automated hearing screening technique for newborns. Acta Otolaryngol (Stockh) 1979;87:1-8.

10 Abramovich SJ, Gregory S, Slemick M, Stewart A. Hearing loss in very low birthweight infants treated with neonatal intensive care. Arch Dis Child 1979;54:421-6.

11 Bhattacharya J, Bennett MJ, Tucker SM. Long term follow up of newborns tested with the auditory response cradle. Arch Dis Child 1984;59:504-11.

12 McCormick B. Hearing screening by health visitors: a critical appraisal of the distraction test. Health Visitor 1983;56:449-51.

13 Bennett MJ, Wade HK. Automated newborn screening using the auditory response cradle. In: Taylor IE, Markides A, eds. Disorders of auditory function III. London: Academic Press, 1980.

14 Davis A. The statistical decision criterion for the auditory response cradle 1984. BrJ Audiol 1984;18:163-8.

15 Martin JAM, Moore WJ. Childhood deafness in the European community. EUR 6413 1979. Luxembourg: Commission of the European Communities, 1979.

16 McCormick B, Wood S, Cope Y, Spavins F. Analysis of records from an open access audiology service. Br J Audiol 1984;18: 127-32.

17 Latham AD, Haggard MP. A pilot study to detect hearingimpairment in the young. Health Visitor, Midwife and Community Nurse 1980;16:370-4.

${ }^{18}$ Mahoney TM, Eichwald JG. Newborn high-risk hearing screening by maternal questionnaire. Journal of the American Auditory Society 1979;5:41-5.

${ }^{19}$ Haggard MP, Wood EJ, Corroll S. Speech, admittance and tone tests in school screening-reconciling economics with pathology_and disability-perspectives. Br J Audiol 1984;18: 133-53.

Correspondence to Dr B McCormick, Hearing Services Centre General Hospital, Nottingham NG1 6HA.

Received 22 August 1984 